\title{
Osteoprotective medication in the era of novel agents: a European perspective on values, risks and future solutions
}

\author{
Monika Engelhardt ${ }^{1,2}$ Georg W. Herget,${ }^{3}$ Giulia Graziani, ${ }^{1,2}$ Gabriele Ihorst,${ }^{4}$ Heike Reinhardt,${ }^{1,2}$ Stefanie Ajayi, ${ }^{1,2}$ \\ Stefan Knop ${ }^{5}$ and Ralph Wasch ${ }^{1,2}$
}

\begin{abstract}
${ }^{1}$ Department of Medicine I, Hematology, Oncology \& Stem Cell Transplantation, Medical Center, University of Freiburg; ${ }^{2}$ Comprehensive Cancer Center Freiburg (CCCF), Medical Center, University of Freiburg; ${ }^{3}$ Department of Orthopedics and Trauma Surgery, Medical Center, University of Freiburg; ${ }^{4}$ Clinical Trials Unit, Medical Center, University of Freiburg and

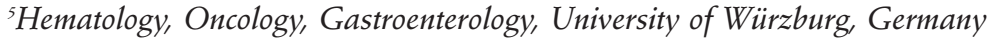

E-mail: monika.engelhardt@uniklinik-freiburg.de
\end{abstract}

doi:10.3324/haematol.2018.188516

O steolytic bone disease is one of the most prominent features of multiple myeloma (MM) and is present in up to $80 \%$ of patients at diagnosis. ${ }^{1}$ Bone destruction leads to skeletal-related events (i.e. vertebral and other pathological fractures) and/or spinal cord compression. MM is mainly due to an increase in osteoclastic activity which is accompanied by low osteoblastic function. ${ }^{1}$ Bisphosphonates and other bone-targeting agents (such as denosumab which inhibits RANKL and osteoclast function and is not renally cleared), effective anti-myeloma treatment, radiotherapy and surgery are the main therapies used for the management of bone disease in $M M{ }^{1-3}$ Regarding the definition of $\mathrm{MM}$-defining events, there are important studies which suggest that asymptomatic patients with more than one focal lesion detectable by magnetic resonance imaging have a higher risk of progression to symptomatic MM (>70\% within 2 years). ${ }^{1,46}$ These patients have been described by international myeloma experts as having symptomatic disease. 5,6

Based on phase 3 studies, the bisphosphonates, pamidronate and zoledronic acid, have been found to reduce skeletal-related events compared to placebo. ${ }^{7-9}$ Three randomized studies have compared the effect of different bisphosphonates or different dosages of the same bisphosphonate. In the first study, zoledronic acid was as effective as pamidronate in reducing skeletal-related events in the era of conventional chemotherapy. ${ }^{9,10}$ In the second, two doses of intravenous pamidronate $(30$ versus $90 \mathrm{mg}$ ) showed comparable results regarding time to skeletal-related events and survival time free of such events. ${ }^{11}$ The limitation of this study was that it was powered to show differences in quality of life and not in skeletal-related events. ${ }^{11}$ The third study compared intravenous (i.v.) zoledronic acid with oral clodronate and showed that zoledronic acid reduced the risk of skeletalrelated events compared to clodronate in all MM patients, irrespective of the presence of lytic lesions at diagnosis, and improved overall survival by 10 months in patients with lytic lesions at diagnosis. ${ }^{12,13}$ These effects continued in patients who received zoledronic acid for $>2$ years. ${ }^{14}$ There was no sub-analysis according to the response status of the patients, thus it is not clear whether the continuous use of zoledronic acid produces similar results in patients who have achieved excellent responses ( $\geq$ very good partial response). A meta-analysis was unable to confirm superiority of zoledronic acid over pamidronate, but revealed a survival advantage from zoledronic acid versus placebo. ${ }^{15}$ This analysis also determined that in order to prevent one skeletal-related event, 6-15 MM patients need to be treated. ${ }^{15}$

The European Myeloma Network (EMN) and International Myeloma Working Group (IMWG) have therefore recommended that all MM patients with adequate renal function (creatinine clearance $>30 \mathrm{~mL} / \mathrm{min}$ ) and osteolytic disease at diagnosis should be treated with zoledronic acid [4 $\mathrm{mg}$ i.v. infusion, over at least $15 \mathrm{~min}$, every 4 weeks (Q4W) or pamidronate $(90 \mathrm{mg}$, in a 3-hour infusion, $\mathrm{Q} 4 \mathrm{~W}]$, in addition to specific anti-myeloma therapy (grade 1A; definition of evidence levels: Online Supplementary Table S1). Symptomatic patients, without bone disease assessed by conventional radiography, can be treated with zoledronic acid (grade 1B). The advantage is not clear for patients without detectable bone involvement on magnetic resonance imaging or positron emission tomography/computed tomography. Bisphosphonates are not routinely recommended in smoldering $\mathrm{MM}$ (grade 1A); but in cases of osteoporosis or vertebral fractures that are not due to the MM, bisphosphonates should be given at the doses given for osteoporosis (5 mg zoledronic acid/year). For high-risk smoldering $\mathrm{MM}$, the treating physician should consider using the bisphosphonate doses and schedules typically used to treat symptomatic MM (grade 1B). Zoledronic acid should be given continuously (grade 1B). It is debatable whether patients who achieve a very good partial response or better have benefits from the continuous use of zoledronic acid. Regarding pamidronate, there are no data to support its continuous use; thus it should be given for 2 years and then at the physician's discretion (grade 2C). ${ }^{1,2}$ Of note, bisphosphonates are now available as generic drugs, whereas denosumab has just been approved by the Food and Drug Administration for use in MM (January 2018; likewise anticipated in Europe) and is patent-protected. This approval was based on the results of a large phase III study comparing denosumab with zoledronic acid, in which the efficacy and safety of the drugs were assessed in newly diagnosed MM. Eligible patients were randomized 1:1 to denosumab $120 \mathrm{mg}$ subcutaneously $\mathrm{Q} 4 \mathrm{~W}$ or zoledronic acid $4 \mathrm{mg}$ (with dose adjustments according to renal function) i.v. Q4W along with anti-myeloma therapy. The primary objective was non-inferiority of denosumab to zoledronic acid with respect to time to first on-study skeletal-related event. Overall survival was a secondary endpoint; progressionfree survival was an exploratory endpoint. The 1718 patients enrolled were randomized into two arms, each 
with 859 participants. With regards to delaying time to first on-study skeletal-related event, denosumab was not inferior to zoledronic acid $[P=0.01$; hazard ratio $(\mathrm{HR})=0.98 ; 95 \%$ confidence interval (95\% CI): 0.85-1.14]. Fewer adverse events potentially related to renal impairment were reported with denosumab than with zoledronic acid $(10.0 \%$ versus $17.1 \%, P<0.001)$. The $\mathrm{HR}$ for progression-free survival was 0.82 (95\% CI: 0.68-0.99; $P=0.036)$. The overall survival $\mathrm{HR}$ between denosumab and zoledronic acid was 0.9 (95\% CI: 0.70-1.16; $P=0.41$ ), with fewer deaths in the denosumab arm $(n=121 ; 14.1 \%)$ than in the zoledronic acid group ( $\mathrm{n}=129 ; 15.0 \%)$. Therefore, denosumab showed non-inferiority to zoledronic acid in delaying time to first on-study skeletalrelated event. Patients on denosumab had a significantly lower rate of renal adverse events compared to those on zoledronic acid. The bone-specific benefits in combination with the renal function results and possible prolongation of progression-free survival with denosumab were promising and have led to invigorating discussions about why progression-free survival data were more favorable with denosumab. The observations definitely need deep-

Table 1. Cost comparison of osteoprotective medications for MM: Germany versus USA.

\begin{tabular}{|c|c|c|c|c|c|}
\hline \multirow[b]{2}{*}{ Drug (original) } & \multirow[b]{2}{*}{ Dose \& mode of administration } & \multicolumn{2}{|c|}{$\begin{array}{c}\text { Costs Germany } \\
\text { [Monthly costs in Euro (€)] }\end{array}$} & \multicolumn{2}{|c|}{$\begin{array}{c}\text { Costs USA } \\
\text { [Monthly costs in Euro }(€)]\end{array}$} \\
\hline & & Original price & Generic price & Original price & Generic price \\
\hline Denosumab $\left(\right.$ Xgeva $\left.^{\circledR}\right)$ & $120 \mathrm{mg}$ s.c. bolus & 440 & - & 1890 & - \\
\hline Zoledronic acid $\left(\right.$ Zometa $\left.^{\circledR}\right)$ & $4 \mathrm{mg}$ i.v. over $15 \mathrm{~min}$ & 368 & 279 & 814 & 49 \\
\hline Pamidronate & $90 \mathrm{mg}$ i.v. over 3 hours & out of trade & 251 & out of trade & 47 \\
\hline
\end{tabular}

German prices: ATaxx ${ }^{\circledast}$ (Dr. Heni Software GmbH \& Co.KG, Freiburg); USA prices: https://www.drugs.com/price-guide/.In Germany, the AMNOG is limiting the cost of new phar maceutical products; In the USA, a deflation of generic prices has been reported, due to an increasing number of competing companies entering the market (https://www.nytimes.com/2017/08/08/health/generic-drugs-prices-falling.html).

A

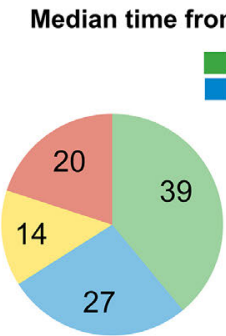

Retrospective analysis $(n=101)$

Median time: 4 months

C
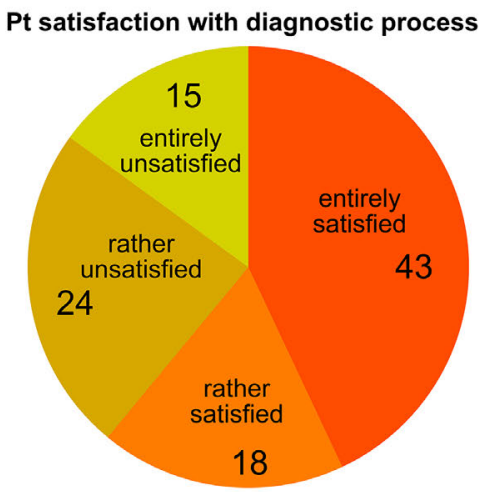

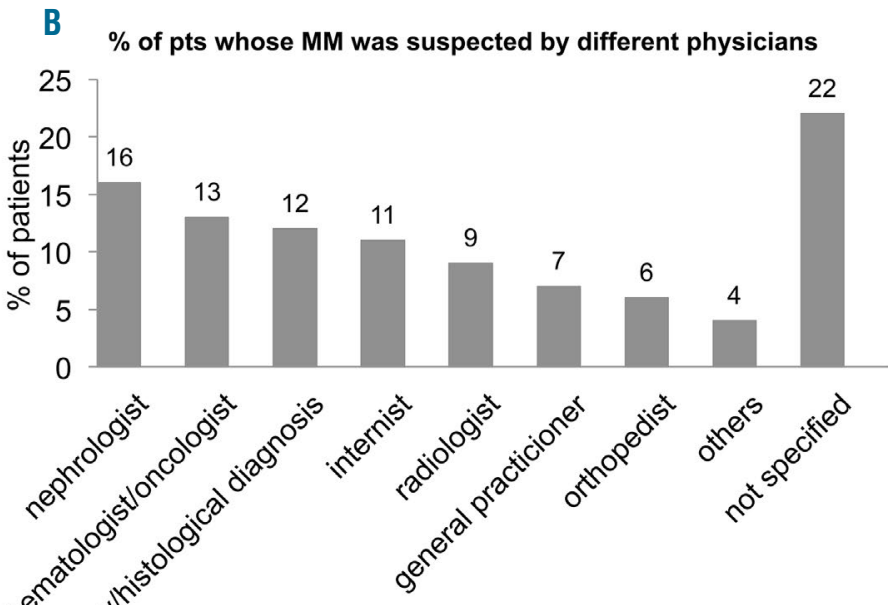

D Pts' estimation, whether their MM could have been diagnosed earlier

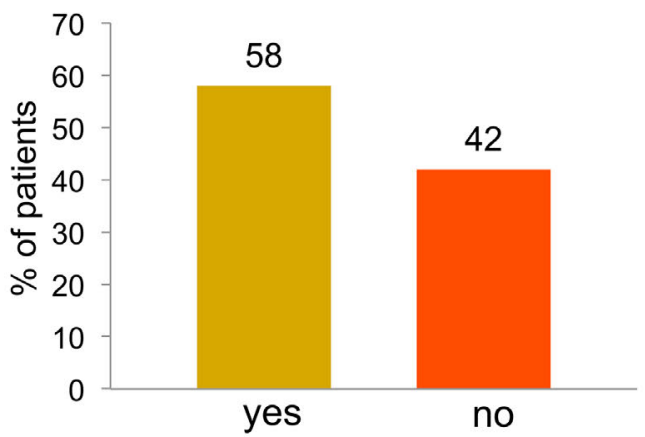

Figure 1. Features of the diagnosis of multiple myeloma. (A) Time from onset of symptoms to the diagnosis of MM: patients (\%) diagnosed within <3, $3-6,6-11$ or $>12$ months in the retrospective versus prospective analysis. (B) First suspicion of MM: frequency (\%) of patients whose MM was first suspected by different types of physicians ( $n=176$ patients; prospective cohort). (C-D) Prospective analysis: patients' satisfaction ( $n=176)$. 
er understanding and statistical evaluation: the relationship between the occurrence of skeletal-related events and progression-free survival-defining events needs to be defined. Furthermore, an assessment of cumulative incidence rates of skeletal-related events with death as a competing event will be helpful, as the slight overall survival disadvantage in the zoledronic acid arm might have led to fewer skeletal-related events. Nevertheless, with full publication of the results ${ }^{16}$ and with EMA approval, denosumab will be used in Europe in MM patients. ${ }^{1-3}$

Given these insights and major advances in the understanding of the disease, early MM diagnosis, especially of symptomatic patients, has been advocated. However, since $M M$ is an insidiously developing malignancy and may appear with non-specific symptoms, e.g. bone pain, the diagnosis and therapeutic decisions can be complicated. A German study group (DSMM) and EMN project addressed this aspect with the aim of optimizing the prompt diagnosis and further improving the quality of MM care. ${ }^{17}$ An initial retrospective analysis of $101 \mathrm{MM}$ patients was followed by a prospective study of 176 patients using a structured MM-specific questionnaire. The median time from the patients' first symptoms to the final MM diagnosis was 4 months (range, 0.5-120) in the retrospectively studied cohort and very similar to the 6 months (range, 0.5-60) in the prospective cohort. Of interest, the time from onset of symptoms to diagnosis of $\mathrm{MM}$ was $\geq 12$ months in $20 \%$ of the patients in the retrospective analysis and $35 \%$ in the prospective study (Figure 1A). The frequencies of $\mathrm{MM}$-related bone fractures, renal complications and infections occurring before the diagnosis of $\mathrm{MM}$ was made were $41 \%, 35 \%$ and $16 \%$, respectively. Moreover, $43 \%$ had one, $20 \%$ had two and $3 \%$ had three of these complications. The most frequent symptom was bone pain, which occurred in $73 \%$ of $\mathrm{MM}$ patients before the final $\mathrm{MM}$ diagnosis was made. In $6 \%$ of patients, MM was first suspected by orthopedists, whereas the clinical suspicion was raised by nephrologists in $16 \%$ of cases, even though renal impairment was less frequent (Figure 1B). Of interest, $61 \%$ of patients were completely or fairly satisfied with the diagnostic process, whereas 39\% were less satisfied (Figure 1C). Fifty-eight percent of the patients believed that their disease could have been diagnosed more expeditiously (Figure 1D). Patients, who criticized the slow diagnostic process had a much longer median time interval from symptom onset to their final MM diagnosis compared to those who were less critical ( 9 versus 3 months, respectively). These results demonstrate that there is still considerable latency in the diagnosis of MM. However, even with early diagnosis and treatment with novel agents, skeletal-related events continue to occur, in part due to $\mathrm{MM}$ responses ("melting-down $\mathrm{MM}^{\prime}$ ) and relapses, reminding us that progress in $\mathrm{MM}$ involves understanding how best to avoid skeletal-related events before the diagnosis of the disease is made and with antimyeloma treatment, because this substantially influences patients' coping and their approval of our MM care. , $3,17^{\text {The notion }}$ that treatment based on novel agents promotes bonehealing - apart from osteoprotective supportive agents such as bisphosphonates and denosumab - has recently led to the demanding discussion ${ }^{18,19}$ of whether bone- seeking agents are currently needed. Since skeletal-related events continue to occur in the first months of treatment and with relapse (despite the use of novel agents and osteoprotection $^{1-3}$ ), effective prevention and reduction of destructive skeletal-related events remain fundamental. ${ }^{1,20}$ Recent data from the national registry, Hospital Episode Statistics determined fracture rates and the effect on overall survival in MM patients between 2001 and 2015: expectedly, fracture rates were 18 times higher with MM in the first year after admission than in the general population, and remained elevated for up to 10 years. In line with the data on early diagnosis in $\mathrm{MM}^{2,3,17}$ the increased fracture risk preceded the first admission with $M M$ and conversely the incidence of $\mathrm{MM}$ increased after admission with one or more fractures. Fractures were associated with poorer outcome (HR for overall survival: 1.2), indicating the need for regular use of bone supportive drugs despite novel agent-based treatment. ${ }^{21}$ In addition, cost analyses in $1028 \mathrm{MM}$ patients (596 with $\geq 1$ skeletalrelated events and 432 without skeletal-related events) demonstrated that a higher frequency of skeletal-related events was associated with greater utilization of healthcare resources, suggesting that bone supportive drugs need to be used diligently to avoid higher healthcare costs due to skeletal complications and patients' discontent. ${ }^{22}$ Since bisphosphonates in symptomatic MM have been suggested, but beyond 2 years and with stable $\mathrm{MM}$ are left to the discretion of the treating physician, a randomized trial assessed 170 untreated, symptomatic patients using zolendronic acid for 4 versus 2 years. ${ }^{23}$ All patients were treated with the same induction therapy and stemcell transplantation. The group treated for 4 years had substantially fewer skeletal-related events than the group treated for 2 years (21 versus $43 \%$, respectively; $P<0.001$ ). Actuarial curves at 5 years showed that progression-free survival was $75 \%$ (95\% CI: $64 \%-82 \%)$ and overall survival $68 \%(95 \% \mathrm{CI}, 60 \%-76 \%)$ in the group treated for 4 years; these rates were not significantly different from those of the control group treated for 2 years with zoledronic acid $(P=0.67)$; but this trial was underpowered to show differences in survival. The trial did, however, confirm that the continued use of zoledronic acid was useful to reduce skeletal-related events and to preserve a better quality of life. $^{23}$

With bisphosphonates and denosumab being potent options in MM, Goldstein, in this issue of the Journal, comments on both costs and the fact that novel patentprotected drugs will induce greater expenditure than generically available alternatives. ${ }^{24}$ While there is an unequivocal need to thoroughly evaluate and measure "real" advances with new drugs, shortcomings of this commentary are the "generalization" regarding patent versus generic medications, the understatement of progression-free survival differences, convenience of subcutaneous versus i.v. medication, and the decreased renal impairment and safer use of denosumab in patients with renal impairment. Moreover, Goldstein's conclusions only apply to the health system in the USA, whereas reimbursement of medication providers and financial incentives to physicians to prescribe more expensive drugs are different in Europe (Table 1). ${ }^{24}$ The rising cost of patented cancer medicines in the USA is a known phe- 
nomenon: from 2000 until now, there have been 5- to 10fold increases in the cost of new drugs. ${ }^{25}$ Price differences in Europe can, therefore, be substantially different from those in the USA (Table 1). In Germany, a benefit assessment of pharmaceuticals in accordance with the Act on the Reform of the Market for Medicinal Products (AMNOG) is limiting the cost of new pharmaceutical products. In the USA, a deflation of generic prices has been reported, due to an increasing number of competing companies entering the market. As clinicians and researchers we know that medical advances are needed, and developmental costs for new drugs have steadily increased with regulatory requirements. Goldstein reminds us of the financial burden associated with new cancer agents, which we offer to our patients with the aim to 'never harm but always aid': nevertheless, the judgement regarding bisphosphonate generics versus denosum$\mathrm{ab}$ is biased and the comparison is cumbersome. His conclusions that generic bisphosphonates have a novel counterpart and that the financial burden with denosumab is higher are, however, worth noting. ${ }^{24}$ Thus, the good news prevails that treatment options for the prevention of bone complications have increased with the introduction of denosumab, providing a new choice for patients and physicians. Once initiated, bisphosphonates or denosum$\mathrm{ab}$ should be continued for at least 2 years, after which a suspension of bisphosphonate treatment may be considered in very responsive patients, although this may be associated with skeletal risks, especially in those with prior skeletal complications. For patients in whom bisphosphonates were stopped after 2 years, the drug should be resumed on a monthly basis if the MM recurs and/or new skeletal-related events occur, independently of the use of novel agent-based therapies. ${ }^{1-3}$

\section{Acknowledgment}

We are deeply indebted to esteemed experts of the Deutsche Studiengruppe Multiples Myelom, German Multiple Myeloma Study Group, European Myeloma Network Group and International Myeloma Working Group for their valuable discussion.

\section{References}

1. Terpos E, Kleber M, Engelhardt M, et al. European Myeloma Network Guidelines for the management of multiple myeloma-related complications. Haematologica. 2015;100(10):1254-1266.

2. Terpos E, Christoulas D, Gavriatopoulou M. Biology and treatment of myeloma related bone disease. Metabolism. 2018;80:80-90.

3. Yee AJ, Raje NS. Denosumab for the treatment of bone disease in solid tumors and multiple myeloma. Future Oncol. 2018;14(3):195203.

4. Hillengass J, Fechtner K, Weber M-A, et al. Prognostic significance of focal lesions in whole-body magnetic resonance imaging in patients with asymptomatic multiple myeloma. J Clin Oncol. 2010;28(9):1606-1610.

5. Rajkumar SV, Dimopoulos MA, Palumbo A, et al. International Myeloma Working Group updated criteria for the diagnosis of multiple myeloma. Lancet Oncol. 2014;15(12):e538-548.

6. Caers J, Fernández de Larrea C, Leleu X, et al. The changing land- scape of smoldering multiple myeloma: a European perspective. Oncologist. 2016;21(3):333-342.

7. Berenson JR, Lichtenstein A, Porter L, et al. Efficacy of pamidronate in reducing skeletal events in patients with advanced multiple myeloma. Myeloma Aredia Study Group. N Engl J Med. 1996;334(8):488-493

8. Berenson JR, Rosen LS, Howell A, et al. Zoledronic acid reduces skeletal-related events in patients with osteolytic metastases. Cancer. 2001;91(7):1191-1200.

9. Rosen LS, Gordon D, Kaminski M, et al. Zoledronic acid versus pamidronate in the treatment of skeletal metastases in patients with breast cancer or osteolytic lesions of multiple myeloma: a phase III, double-blind, comparative trial. Cancer J. 2001;7(5): 377-387.

10. Rosen LS, Gordon D, Kaminski M, et al. Long-term efficacy and safety of zoledronic acid compared with pamidronate disodium in the treatment of skeletal complications in patients with advanced multiple myeloma or breast carcinoma: a randomized, double-blind, multicenter, comparative trial. Cancer. 2003;98(8):1735-1744.

11. Gimsing P, Carlson K, Turesson I, et al. Effect of pamidronate $30 \mathrm{mg}$ versus $90 \mathrm{mg}$ on physical function in patients with newly diagnosed multiple myeloma (Nordic Myeloma Study Group): a double-blind, randomised controlled trial. Lancet Oncol. 2010;11(10):973-982.

12. Morgan GJ, Davies FE, Gregory WM, et al. First-line treatment with zoledronic acid as compared with clodronic acid in multiple myeloma (MRC Myeloma IX): a randomised controlled trial. Lancet. 2010;376(9757):1989-1999

13. Morgan GJ, Child JA, Gregory WM, et al. Effects of zoledronic acid versus clodronic acid on skeletal morbidity in patients with newly diagnosed multiple myeloma (MRC Myeloma IX): secondary outcomes from a randomised controlled trial. Lancet Oncol. 2011;12(8):743-752.

14. Morgan GJ, Davies FE, Gregory WM, et al. Effects of induction and maintenance plus long-term bisphosphonates on bone disease in patients with multiple myeloma: the Medical Research Council Myeloma IX Trial. Blood. 2012;119(23):5374-5383.

15. Mhaskar R, Redzepovic J, Wheatley K, et al. Bisphosphonates in multiple myeloma: a network meta-analysis. Cochrane Database Syst Rev. 2012;(5):CD003188.

16. Raje N, Terpos E, Willenbacher W, et al. An international, randomised, double-blind study of denosumab compared to zoledronic acid in bone disease treatment of newly diagnosed multiple myeloma. Lancet Hematol. 2018;19(3):370-381.

17. Graziani G, Herget G, Ihorst G, et al. Time from first symptom onset to the final diagnosis of multiple myeloma - possible risks and future solutions: large retrospective and conformatory prospective "Deutsche Studiengruppe Multiples Myelom" (DSMM) analysis. Blood. 2017;130(Suppl 1):4710.

18. Delforge M, Terpos E, Richardson PG, et al. Fewer bone disease events, improvement in bone remodeling, and evidence of bone healing with bortezomib plus melphalan-prednisone vs. melphalanprednisone in the phase III VISTA trial in multiple myeloma. Eur J Haematol. 2011;86(5):372-384

19. Mohty M, Malard F, Mohty B, Savani B, Moreau P, Terpos E. The effects of bortezomib on bone disease in patients with multiple myeloma. Cancer. 2014;120(5):618-623.

20. Engelhardt M, Terpos E, Kleber M, et al. European Myeloma Network recommendations on the evaluation and treatment of newly diagnosed patients with multiple myeloma. Haematologica. 2014;99(2):232-242.

21. Mcllroy G, Mytton J, Evison F, et al. Increased fracture risk in plasma cell dyscrasias is associated with poorer overall survival. Br J Haematol. 2017;179(1):61-65.

22. Nash Smyth E, Conti I, Wooldridge JE, et al. Frequency of skeletalrelated events and associated healthcare resource use and costs in US patients with multiple myeloma. J Med Econ. 2016;19(5):477-486.

23. Avilès $\mathrm{A}, \mathrm{Nambo} \mathrm{M}-\mathrm{J}$, Huerta-Guzmàn J, Cleto $\mathrm{S}$, Neri N. Prolonged use of zoledronic acid (4 Years) did not improve outcome in multiple myeloma patients. Clin Lymphoma Myeloma Leuk. 2017;17(4):207210

24. Goldstein D. Denosumab for bone lesions in multiple myeloma what is its value? Haematologica. 2018(5):753-754.

25. Kantarjian H, Steensma D, Rius Sanjuan J, Elshaug A, Light D. High cancer drug prices in the United States: reasons and proposed solutions. J Oncol Pract. 2014;10(4):e208-211. 